\title{
ON THE REFLEXIVITY AND HYPERREFLEXIVITY OF ALGEBRAS AND SUBSPACES
}

\author{
MAREK PTAK \\ Institute of Mathematics, University of Agriculture \\ Al. Mickiewicza 24/28, 30-059 Kraków, Poland \\ E-mail: rmptak@cyf-kr.edu.pl
}

\begin{abstract}
A review of recent reflexivity and hyperreflexivity results is presented. We concentrate particularly on a finite-dimensional situation, Toeplitz operators and partial isometries. Open problems in this area are given.
\end{abstract}

1. Introduction. Let $\mathcal{H}$ be a complex Hilbert space and let $L(\mathcal{H})$ denote the algebra of all bounded linear operators on $\mathcal{H}$. Consider a subalgebra $\mathcal{W}$ of $L(\mathcal{H})$ with an identity $I$. Then we can investigate the lattice of invariant subspaces:

$$
\text { Lat } \mathcal{W}=\{\mathcal{L} \subset \mathcal{H}: A \mathcal{L} \subset \mathcal{L} \text { for all } A \in \mathcal{W}\}
$$

Now the set of those operators which leave invariant all subspaces from Lat $\mathcal{W}$ can be defined:

$$
\operatorname{Alg} \text { Lat } \mathcal{W}=\{B \subset L(\mathcal{H}): B \mathcal{L} \subset \mathcal{L}, \mathcal{L} \in \text { Lat } \mathcal{W}\}
$$

The set Alg Lat $\mathcal{W}$ is always an algebra which is closed in the weak operator topology and which contains the identity $I_{\mathcal{H}}$. There are the following inclusions:

$$
\mathcal{W} \subset \operatorname{Alg} \operatorname{Lat} \mathcal{W} \subset L(\mathcal{H}) .
$$

In general the above inclusions may be proper.

Following [34] we call the algebra $\mathcal{W}$ reflexive if $\mathcal{W}=\operatorname{Alg}$ Lat $\mathcal{W}$, i.e., the first inclusion becomes an equality. The algebra is said to be transitive if $\operatorname{Alg} \operatorname{Lat} \mathcal{W}=L(\mathcal{H})$, i.e., the second inclusion becomes an equality. The classical definition of a transitive algebra requires that Lat $\mathcal{W}=\{\mathcal{H},\{0\}\}$, but it is equivalent to the condition $\operatorname{Alg} \operatorname{Lat} \mathcal{W}=L(\mathcal{H})$.

2000 Mathematics Subject Classification: Primary 47L05; Secondary 47L80, 47A15, 47L45.

Key words and phrases: reflexive subspaces, hyperreflexive subspace, hyperreflexive constant, $k$-hyperreflexive subspace, Toeplitz operator, partial isometry, power partial isometry.

The paper is supported by grant No 1 P03A 01627 of KBN, Poland.

The paper is in final form and no version of it will be published elsewhere. 
The theory of reflexive algebras has its roots in von Neumann algebras. They are defined as star algebras which are closed in the strong (weak) operator topology. For such an algebra $\mathcal{N}$ we consider the commutant $\mathcal{N}^{\prime}$. Recall first that the projections in $\mathcal{N}^{\prime}$ generate $\mathcal{N}^{\prime}$. The projections in $\mathcal{N}^{\prime}$ commute with all operators from $\mathcal{N}$, i.e., the subspaces which are ranges of such projections reduce all the operators in $\mathcal{N}$. When we take an unstarred algebra $\mathcal{W}$ it is natural to consider invariant subspaces in Lat $\mathcal{W}$ instead of reducing ones. For a von Neumann algebra we consider also its double commutant $\mathcal{N}^{\prime \prime}$. Since the commutant $\mathcal{N}^{\prime}$ is generated by its projections the double commutant can be defined as the set of those operators which are reduced by those subspaces which reduce all the operators from $\mathcal{N}$. Hence for an unstarred algebra $\mathcal{W}$ it is natural to consider Alg Lat $\mathcal{W}$ instead of its double commutant. The classical double commutant theorem says that a von Neumann algebra $\mathcal{N}$ is equal to its double commutant. Hence the reflexive algebras are those which fulfil an "unstarred version of the double commutant theorem".

For a given algebra (or a subspace) $\mathcal{W} \subset L(\mathcal{H})$ and an operator $A \in L(\mathcal{H})$ the usual distance from $A$ to $\mathcal{W}$ is given as

$$
\operatorname{dist}(A, \mathcal{W})=\inf \{\|A-T\|: T \in \mathcal{W}\} .
$$

On the other hand, we can define the distance "determined by its invariant subspaces" as

$$
\alpha(A, \mathcal{W})=\sup \left\{\left\|P^{\perp} A P\right\|: P \in \operatorname{Lat} \mathcal{W}\right\} .
$$

We have the following inequality

$$
\alpha(A, \mathcal{W}) \leqslant \operatorname{dist}(A, \mathcal{W}) \quad \text { for all } A \in L(H) .
$$

Indeed, for $T \in \mathcal{W}, P \in$ Lat $\mathcal{W}$ and any operator $A \in L(\mathcal{H})$ we have

$$
\left\|P^{\perp} A P\right\|=\left\|P^{\perp}(A-T) P\right\| \leqslant\|A-T\| .
$$

Hence $\alpha(A, \mathcal{W}) \leqslant\|A-T\|$ and (4) holds.

Usually we do not have an equality in (4). A natural question is whether we can control the usual distance dist by the $\alpha$ distance. Arveson [3] called an algebra hyperreflexive if there is a constant $k$ such that for all $A \in L(\mathcal{H})$

$$
\operatorname{dist}(A, \mathcal{W}) \leqslant k \alpha(A, \mathcal{W}) .
$$

Reflexivity and hyperreflexivity are also studied when the operators which generate an algebra are given. For a set of operators $\mathcal{S} \subset L(\mathcal{H})$ we denote by $\mathcal{W}(\mathcal{S})$ the smallest algebra containing the set $\mathcal{S}$ and the identity operator $I$ which is closed in the weak operator topology.

2. Definitions for subspaces of operators. Both definitions, reflexivity and hyperreflexivity, can be generalized to subspaces of operators. Let $\mathcal{M} \subset L(\mathcal{H})$ be a subspace. The set

$$
\operatorname{ref} \mathcal{M}=\left\{B \in L(\mathcal{H}): B x \in[\mathcal{M} x]^{-} \text {for all } x \in \mathcal{H}\right\}
$$

is called the reflexive closure of $\mathcal{M}$. If $\mathcal{M}$ is an algebra with identity $I$, then ref $\mathcal{M}=$ Alg Lat $\mathcal{M}$. 
Note also that we have similar inclusion as in (1)

$$
\mathcal{M} \subset \operatorname{ref} \mathcal{M} \subset L(\mathcal{H}) .
$$

Following Loginov and Shulman [28] we will call a subspace $\mathcal{M}$ reflexive if $\mathcal{M}=\operatorname{ref} \mathcal{M}$ and $\mathcal{M}$ is transitive if the second inclusion in (7) becomes an equality, i.e., ref $\mathcal{M}=L(\mathcal{H})$.

For a subspace $\mathcal{M}$ and an operator $A \in L(\mathcal{H})$ the usual distance $\operatorname{dist}(A, \mathcal{M})$ is naturally given by (2). The $\alpha$ distance can be defined as follows (see [27])

$$
\alpha(A, \mathcal{M})=\sup \left\{\left\|Q^{\perp} A P\right\|: Q^{\perp} \mathcal{M} P=0, Q, P \text { projections }\right\} .
$$

A subspace $\mathcal{M}$ is called hyperreflexive if there is a constant $k$ such that

$$
\operatorname{dist}(A, \mathcal{M}) \leqslant k \alpha(A, \mathcal{M}) \text { for all } A \in L(\mathcal{H}) \text {. }
$$

The smallest constant $k$ fulfilling (9) is called constant of hyperreflexivity and is denoted by $k_{\mathcal{M}}$.

As we have seen above there is no need to have an algebra structure in order to define reflexivity and hyperreflexivity. When a subspace of operators is an algebra then the definitions coincide. On the other hand, given a subspace $\mathcal{M} \subset L(\mathcal{H})$ we can define an algebra associated with this subspace by the natural embedding

$$
\mathcal{W}_{\mathcal{M}}=\left\{\left[\begin{array}{cc}
\alpha I & T \\
0 & \beta I
\end{array}\right]: T \in \mathcal{M}, \alpha, \beta \in \mathbb{C}\right\} .
$$

Then we have the following

TheOREM 2.1 ([8, 27]). Let $\mathcal{M} \subset L(\mathcal{H})$ be a subspace of operators. Then $\mathcal{M}$ is reflexive (hyperreflexive) if and only if the algebra $\mathcal{W}_{\mathcal{M}}$ is reflexive (hyperreflexive).

3. Duality. A duality between the trace class operators $\tau c(\mathcal{H})$ and the algebra $L(\mathcal{H})$ is given by

$$
\langle A, t\rangle=\operatorname{tr}(A t) \quad \text { for } A \in L(\mathcal{H}), t \in \tau c(\mathcal{H}) .
$$

An important role in reflexivity and hyperreflexivity is played by rank one operators; for $x, y \in \mathcal{H}$ we define $(x \otimes y) z=(z, y) x$ for $z \in \mathcal{H}$. The action of a rank one operator $x \otimes y$ on any operator $A \in L(\mathcal{H})$ can be expressed as

$$
\langle A, x \otimes y\rangle=\operatorname{tr}(A(x \otimes y))=(A x, y) .
$$

Let $\mathcal{M} \subset L(\mathcal{H})$ be a subspace, then we denote the preannihilator of $\mathcal{M}$ by $\perp \mathcal{M} \subset \tau c(\mathcal{H})$ and the unit ball in $\perp \mathcal{M}$ by ball $\perp \mathcal{M}$. Reflexivity and transitivity can be described by rank one operators. It is easy to note that a subspace is transitive if there is no rank one operator (except the zero operator) in its preannihilator and a $w^{*}$-closed subspace is reflexive if the rank one operators in the preannihilator span the preannihilator.

Assume that a subspace $\mathcal{M} \subset L(\mathcal{H})$ is $w^{*}$-closed. Then, by duality, the distance dist can be calculated by means of trace class operators, i.e.

$$
\operatorname{dist}(A, \mathcal{M})=\sup \left\{|\langle A, t\rangle|: t \in \tau c, t \in \operatorname{ball}_{\perp} \mathcal{M}\right\} \quad \text { for all } A \in L(\mathcal{H}) .
$$

On the other hand, the distance $\alpha$ can be calculated using only rank one operators, see [14]. Namely

$$
\alpha(A, \mathcal{M})=\sup \left\{|(A x, y)|=|\langle A, x \otimes y\rangle|: x \otimes y \in \operatorname{ball}_{\perp} \mathcal{M}\right\} .
$$


Now the inequality

$$
\alpha(A, \mathcal{M}) \leqslant \operatorname{dist}(A, \mathcal{M})
$$

is more transparent. Hence hyperreflexivity of a subspace $\mathcal{M}$ means that the distance from an operator to $\mathcal{M}$ can be controlled by the distance calculated using rank one operators. The distance $\alpha$ can also be calculated as

$$
\alpha(A, \mathcal{M})=\sup \{\operatorname{dist}(A x, \mathcal{M} x): x \in \mathcal{H},\|x\|=1\} .
$$

The reason why we call a subspace hyperreflexive can be seen by the following.

Proposition 3.1. Let $\mathcal{M} \subset L(\mathcal{H})$ be a norm-closed subspace. If $\mathcal{M}$ is hyperreflexive then $\mathcal{M}$ is reflexive.

Proof. Assume that $A \in \operatorname{ref} \mathcal{M}$. Using (10) it is clear that $\alpha(A, \mathcal{M})=0$. Thus by hyperreflexivity $\operatorname{dist}(A, \mathcal{M})=0$ and by norm-closedness $A \in \mathcal{M}$.

The inverse implication is not true.

EXAMPLE 3.2. To construct a subspace which is reflexive, but not hyperreflexive, it is enough to take a sequence of hyperreflexive subspaces $\mathcal{M}_{n} \subset L\left(\mathcal{H}_{n}\right)$ with unboundedly increasing constants of hyperreflexivity (see Example 5.2). We can do this even if each underlying Hilbert space $\mathcal{H}_{n}$ is two-dimensional. Then we take $\mathcal{M}=\bigoplus_{n=1}^{\infty} \mathcal{M}_{n}$ and it is not hard to show that $\mathcal{M}$ is reflexive, but not hyperreflexive.

4. The finite-dimensional case. If the underlying Hilbert space is finite-dimensional then reflexivity and hyperreflexivity are equivalent.

TheOREM 4.1. Let $\mathcal{H}$ be a finite-dimensional Hilbert space. If $\mathcal{M} \subset L(\mathcal{H})$ then $\mathcal{M}$ is reflexive if and only if $\mathcal{M}$ is hyperreflexive.

The theorem above is clear since $\operatorname{dim} L(\mathcal{H}) / \mathcal{M}<\infty$. Indeed, since $\mathcal{M}$ is reflexive then

$$
T+\mathcal{M} \mapsto \alpha(T, \mathcal{M})
$$

and

$$
T+\mathcal{M} \mapsto \operatorname{dist}(T, \mathcal{M})
$$

are norms on $L(\mathcal{H}) / \mathcal{M}$. Since $L(\mathcal{H}) / \mathcal{M}$ is finite-dimensional all the norms are equivalent and we get hyperreflexivity of $\mathcal{M}$.

The notion of reflexivity is interesting even in the finite-dimensional case.

EXAmple 4.2. The algebra $\mathcal{W}_{1}=\left\{\left[\begin{array}{ll}\alpha & \beta \\ 0 & \alpha\end{array}\right]: \alpha, \beta \in \mathbb{C}\right\}$ is not reflexive since Lat $\mathcal{W}_{1}=$ $\{\mathbb{C} \oplus \mathbb{C}, \mathbb{C} \oplus\{0\},\{0\}\}$ and Alg Lat $\mathcal{W}_{1}=\left\{\left[\begin{array}{ll}\alpha & \beta \\ 0 & \gamma\end{array}\right]: \alpha, \beta, \gamma \in \mathbb{C}\right\}$.

On the other hand the algebra $\mathcal{W}_{2}=\left\{\left[\begin{array}{ll}\alpha & \beta \\ 0 & \alpha\end{array}\right] \oplus[\alpha]: \alpha, \beta \in \mathbb{C}\right\}$ is reflexive. This can easily be verified if we note that the following subspaces are in Lat $\mathcal{W}_{2}: \mathbb{C}^{2} \oplus\{0\},\{0\} \oplus$ $\{0\} \oplus \mathbb{C}, \mathbb{C} \oplus\{0\} \oplus\{0\},\{(x, 0, x): x \in \mathbb{C}\},\{(0, x, x): x \in \mathbb{C}\}$. 
Note that both algebras are simply generated and

$$
\mathcal{W}_{1}=\mathcal{W}\left(\left[\begin{array}{ll}
0 & 1 \\
0 & 0
\end{array}\right]\right), \quad \mathcal{W}_{2}=\mathcal{W}\left(\left[\begin{array}{ll}
0 & 1 \\
0 & 0
\end{array}\right] \oplus[0]\right)
$$

Deddens and Fillmore [20] gave a full characterization when an algebra generated by a nilpotent in a finite-dimensional space is reflexive.

THEOREM 4.3 ([20]). Let $T$ be a nilpotent in a finite-dimensional Hilbert space. The algebra $\mathcal{W}(T)$ is reflexive if and only if the sizes of the two largest blocks in the Jordan decomposition differ by no more than one.

Analysis of the above condition leads us to a connection between block sizes and operators of rank two.

REMARK. The algebra $\mathcal{W}(T)$ is reflexive if and only if each rank two operator $A \in \mathcal{W}(T)$ generates a one-dimensional ideal (i.e., $\operatorname{dim} A \mathcal{W}(T) \leqslant 1$ ).

One can ask about characterizations for algebras generated by more than one operator. In [7] families of doubly commuting nilpotent operators were considered. It turns out that the same characterization also works for more general situations.

THEOREM 4.4 ([7]). Suppose $\mathcal{W}$ is an operator algebra generated by a family of doubly commuting nilpotents. Then, in order for $\mathcal{W}$ to be reflexive, it is necessary and sufficient that each rank two member of $\mathcal{W}$ generates a one-dimensional ideal.

REMARK. It is also possible to give a necessary and sufficient condition for reflexivity of a family of doubly commuting nilpotents in finite dimensional Hilbert space using the sizes of blocks (see [7]).

5. Finite-dimensional subspaces of operators. Now one can ask whether reflexivity and hyperreflexivity are equivalent if the operators act on infinite-dimensional Hilbert space but the dimension of the subspace of operators is finite. The problem was stated by Larson and Kraus in [27].

In [4] it was shown that one-dimensional subspaces are always reflexive. Hyperreflexivity of one-dimensional spaces was shown in [27] with a constant of hyperreflexivity smaller than 4 . The optimal constant 1 was found in [29]. A first approach to hyperreflexivity of one-dimensional subspaces was carried out in [2], where hyperreflexivity of the subspace generated by the identity operator was presented.

A positive answer for the problem of Larson and Kraus was given in [30]. In fact it was obtained in the more general setting of Banach spaces.

Let $X, Y$ be Banach spaces and let $L(X, Y)$ denote the algebra of all bounded linear operators from $X$ to $Y$. Let $\mathcal{M}$ be a subspace of $L(X, Y)$. The reflexive closure of $\mathcal{M}$ can be defined as in (6)

$$
\operatorname{ref} \mathcal{M}=\left\{B \in L(X, Y): B x \in[\mathcal{M} x]^{-} \text {for all } x \in X\right\}
$$

and $\mathcal{M}$ is called reflexive if and only if $\mathcal{M}=\operatorname{ref} \mathcal{M}$.

Let $A \in L(X, Y)$. We recall that

$$
\operatorname{dist}(A, \mathcal{M})=\inf \{\|A-T\|: T \in \mathcal{M}\} .
$$


To define the $\alpha$ distance in the Banach space case we can use the formula (11):

$$
\alpha(A, \mathcal{M})=\sup \{\operatorname{dist}(A x, \mathcal{M} x): x \in X,\|x\|=1\} .
$$

Similarly $\mathcal{M}$ is called hyperreflexive if there is a constant $k$ such that

$$
\operatorname{dist}(A, \mathcal{M}) \leqslant k \alpha(A, \mathcal{M}) \text {. }
$$

Theorem 5.1 ([30]). Let $X, Y$ be Banach spaces and let $\mathcal{M} \subset L(X, Y)$ be a linear subspace with $\operatorname{dim} \mathcal{M}<\infty$. Then $\mathcal{M}$ is hyperreflexive if and only if $\mathcal{M}$ is reflexive.

ExAmPLE 5.2. Since each reflexive finite-dimensional subspace of operators is hyperreflexive, one might expect that the hyperreflexivity constant can depend only on the dimension of the subspace. This is not true. Following [30] define $\mathcal{M}_{n}=\left\{\left[\begin{array}{ll}\alpha & \beta \\ 0 & \alpha\end{array}\right] \oplus\left[\frac{1}{n} \alpha\right]\right.$ : $\alpha, \beta \in \mathbb{C}\}$. Then $\operatorname{dim} \mathcal{M}_{n}=2$ and $\mathcal{M}_{n}$ is reflexive thus hyperreflexive, but the hyperreflexivity constant satisfies $k_{\mathcal{M}_{n}}>\frac{n}{2}$. A different example was given in [27]: If $\mathcal{M}_{n}=\left\{\left[\begin{array}{cc}0 & \alpha \\ \beta & -n(\alpha+\beta)\end{array}\right]: \alpha, \beta \in \mathbb{C}\right\}$, then $\mathcal{M}_{n}$ is reflexive and $k_{\mathcal{M}_{n}} \geqslant \frac{n}{3}$.

6. Toeplitz operators. Let $H^{2}$ and $H^{\infty}$ denote the classical Hardy spaces on the unit disc $\mathbb{D}$. We denote the unit circle by $\mathbb{T}$ and we write $L^{2}$ instead of $L^{2}(\mathbb{T}, m)$. We denote the projection from $L^{2}$ onto $H^{2}$ by $P_{H^{2}}: L^{2} \rightarrow H^{2}$. The unilateral shift can be seen as an operator $S \in L\left(H^{2}\right),(S f) z=z f(z)$ for $f \in H^{2}$.

For a given function $\varphi \in L^{\infty}$ we can define a Toeplitz operator with the symbol $\varphi$ as

$$
T_{\varphi} f=P_{H^{2}}(\varphi f) \quad \text { for } f \in H^{2} .
$$

We will denote by $\mathcal{A}=\left\{T_{\varphi}: \varphi \in H^{\infty}\right\}$ the set of all analytic Toeplitz operators. This is an algebra, and indeed $\mathcal{A}=\mathcal{W}(S)$. We denote the space of all Toeplitz operators by $\mathcal{T}=\left\{T_{\varphi}: \varphi \in L^{\infty}\right\}$

The first reflexivity result concerning Toeplitz operators was shown by Sarason.

TheOREM 6.1 ([34]). If $S$ is the unilateral shift then $\mathcal{W}(S)$ is reflexive.

Proof. Note that the vectors $k_{\lambda}=\frac{1}{1-\lambda z}, \lambda \in \mathbb{D}$, are eigenvectors of the backward shift (i.e. $S^{*} k_{\lambda}=\lambda k_{\lambda}$ ). Moreover, the set $\left\{k_{\lambda}: \lambda \in \mathbb{D}\right\}$ is linearly dense in the Hardy space $H^{2}$.

Now when we take an operator $A \in \operatorname{Alg} \operatorname{Lat} \mathcal{W}(S)=\operatorname{Alg} \operatorname{Lat} S$, then $A^{*} \in \operatorname{Alg} \operatorname{Lat} S^{*}$. Since the one-dimensional subspace $\mathbb{C} k_{\lambda}$ belongs to Lat $A^{*}$ the operator $A^{*}$ commutes with $S^{*}$ on $\mathbb{C} k_{\lambda}$ for $\lambda \in \mathbb{D}$. By the density of $\left\{k_{\lambda}: \lambda \in \mathbb{D}\right\}$ in $H^{2}$ we get commutativity of $A^{*}$ and $S^{*}$. Hence $S$ and $A$ commute and by the description of the commutant of $S$ we get that $A \in \mathcal{W}(S)$.

Davidson improved this result.

TheOREm 6.2 ([17]). Let $S$ be the unilateral shift. Then $\mathcal{W}(S)$ is hyperreflexive and $k_{\mathcal{W}(S)}$ $<19$.

There was an improvement of the hyperreflexivity constant in $[25], k_{\mathcal{W}(S)}<13$. The proof of this theorem is not as elementary as in the reflexive case. Nehari's theorem is 
strongly used. It says that

$$
\left\|H_{\varphi}\right\|=\operatorname{dist}\left(\varphi, H^{\infty}\right) \quad \text { for } \varphi \in L^{\infty},
$$

where $H_{\varphi} \in L\left(H^{2}, L^{2} \ominus H^{2}\right)$ is the Hankel operator defined as $H_{\varphi} f=P_{L^{2} \ominus H^{2}}(\varphi f)$ for $f \in H^{2}$.

In general reflexivity and hyperreflexivity are not hereditary, but, in this case, using property $\mathbb{A}$ and $\mathbb{A}_{1}(1)$ (see [14]), it is not hard to show that

Proposition 6.3 ([34]). Let $\mathcal{M} \subset \mathcal{A}$ be a $w^{*}$-closed subspace. Then $\mathcal{M}$ is reflexive and hyperreflexive.

On the other hand

Proposition 6.4 ([6]). The space of all Toeplitz operators $\mathcal{T}$ is transitive.

Proof. Let $f, g \in H^{2}$ and $f \otimes g \in \perp \mathcal{T}$. Then, for all $\varphi \in L^{\infty}$, we have

$$
0=\left\langle T_{\varphi}, f \otimes g\right\rangle=\left(T_{\varphi} f, g\right)=\int \varphi f \bar{g} d m
$$

Since this holds for all functions $\varphi \in L^{\infty}$, it follows that $f \bar{g}=0$ as a function in $L^{1}$. Since $f, g \in H^{2}$ both functions $f, g$ cannot be equal to 0 on a set of positive measure in $\mathbb{T}$ unless they are zero. Hence there is no rank one operator in $\perp \mathcal{T}$ and $\mathcal{T}$ is transitive.

The following dichotomy result between transitivity and reflexivity of Toeplitz operators holds.

TheOREm 6.5 ([6]). Let $\mathcal{B} \subset \mathcal{T}$ be a $w^{*}$-closed subspace. Then the following are equivalent:

1. $\mathcal{B}$ is reflexive,

2. $\mathcal{B}$ is not transitive,

3. there exists $f \in L^{1}$ such that $\log |f| \in L^{1}$ and $\int f g d m=0$ for all $g \in \mathcal{B}$.

The conditions 1 and 2 give the dichotomy while the condition 3 gives a full characterization of reflexive subspaces of Toeplitz operators. There is also an extension of Theorem 6.3 .

Theorem 6.6 ([6]). Let $\mathcal{B}$ be a $w^{*}$-closed algebra such that $\mathcal{A} \subset \mathcal{B} \varsubsetneqq \mathcal{T}$. Then $\mathcal{B}$ is reflexive.

As a consequence of the above we obtain the following two results.

EXAMPLE 6.7. The subspace

$$
S^{* n} \mathcal{A}=\operatorname{span}\left\{S^{* n}, S^{* n-1}, \ldots, S^{*}, I, S, S^{2}, \ldots\right\}
$$

is reflexive.

ExAmPle 6.8. Let $E \subset \mathbb{T}$ such that $0<m(E)<1$ and let $\chi_{E}$ denote the characteristic function of the set $E$. Then $T_{\chi_{E}} \mathcal{A}$ is not reflexive.

Comparing the above results the following problem arises:

Problem 6.9. Which reflexive subspaces of $\mathcal{T}$ are hyperreflexive?

It is worthwhile to add that, in the context of Theorem 6.5, the Bergman shift was investigated in [15] and generalized Toeplitz operators were considered in [24]. 
7. Toeplitz operators on the bidisc and the unit ball. We can also consider Hardy spaces $H^{2}\left(\mathbb{D}^{2}\right), H^{\infty}\left(\mathbb{D}^{2}\right)$ on the bidisc $\mathbb{D}^{2}$ and the projection $P_{H^{2}\left(\mathbb{D}^{2}\right)}: L^{2}\left(\mathbb{T}^{2}\right) \rightarrow H^{2}\left(\mathbb{D}^{2}\right)$. For $\varphi \in L^{\infty}\left(\mathbb{T}^{2}\right)$ we define the Toeplitz operator with symbol $\varphi$ as

$$
T_{\varphi} f=P_{H^{2}\left(\mathbb{D}^{2}\right)}(\varphi f) \quad \text { for } f \in H^{2}\left(\mathbb{D}^{2}\right) .
$$

Then the multiplication operators by the independent variables can be written as

$$
\left(T_{z_{i}} f\right)\left(z_{1}, z_{2}\right)=z_{i} f\left(z_{1}, z_{2}\right) \quad \text { for } f \in H^{2}\left(\mathbb{D}^{2}\right), i=1,2 .
$$

We denote the space of all Toeplitz operators by $\mathcal{T}\left(\mathbb{D}^{2}\right)$ and the algebra of analytic Toeplitz operators by $\mathcal{A}\left(\mathbb{D}^{2}\right)=\left\{T_{\varphi}: \varphi \in H^{\infty}\left(\mathbb{D}^{2}\right)\right\}$, which is equal to $\mathcal{W}\left(T_{z_{1}}, T_{z_{2}}\right)$.

Theorem 6.1 and Proposition 6.4 can be generalized to the bidisc situation.

Theorem 7.1 ([31, 33]).

1. The algebra $\mathcal{A}\left(\mathbb{D}^{2}\right)=\mathcal{W}\left(T_{z_{1}}, T_{z_{2}}\right)$ is reflexive.

2. The subspace $\mathcal{T}\left(\mathbb{D}^{2}\right)$ is transitive.

The proof of reflexivity of $\mathcal{A}\left(\mathbb{D}^{2}\right)$ is similar to the disc case. Note that the set $\left\{k_{\lambda_{1}, \lambda_{2}}=\frac{1}{1-\lambda_{1} z_{1}} \frac{1}{1-\lambda_{2} z_{2}}: \lambda_{1}, \lambda_{2} \in \mathbb{D}\right\}$ is dense in $H^{2}\left(\mathbb{D}^{2}\right)$ and that the function $k_{\lambda_{1}, \lambda_{2}}$ is an eigenvector for $T_{z_{i}}, i=1,2$.

Thus the following problem arises:

Problem 7.2. How can one characterize the reflexive subspaces of $\mathcal{T}\left(\mathbb{D}^{2}\right)$ ?

In the context of Theorem 6.2 we can ask about the hyperreflexivity of $\mathcal{A}\left(\mathbb{D}^{2}\right)$. As we have noticed, one of the tools for the proof of hyperreflexivity of $\mathcal{A}$ was Nehari's Theorem.

In [9], [22] it was shown that Nehari's Theorem cannot be extended to the case of the bidisc. Thus we have the following problem.

Problem 7.3. Is $\mathcal{A}\left(\mathbb{D}^{2}\right)$ hyperreflexive?

The bidisc $\mathbb{D}^{2}$ is not the only possible multivariable generalization of the disc $\mathbb{D}$, and the two-dimensional unit ball $\mathbb{B}^{2}$ is equally significant.

Let $H^{2}\left(\mathbb{B}^{2}\right)$ and $H^{\infty}\left(\mathbb{B}^{2}\right)$ be the Hardy spaces on the ball $\mathbb{B}^{2}$. Let $P_{H^{2}\left(\mathbb{B}^{2}\right)}: L^{2}\left(\partial \mathbb{B}^{2}\right) \rightarrow$ $H^{2}\left(\mathbb{B}^{2}\right)$ be the projection. We can define Toeplitz operators similarly to the case of the disc. For $\varphi \in L^{\infty}\left(\partial \mathbb{B}^{2}\right)$ we define an operator $T_{\varphi}$ by $\left(T_{\varphi} f\right)=P_{H^{2}\left(\mathbb{B}^{2}\right)}(\varphi f)$ for $f \in H^{2}\left(\mathbb{B}^{2}\right)$. We keep the notations $\mathcal{A}\left(\mathbb{B}^{2}\right)$ for analytic and $\mathcal{T}\left(\mathbb{B}^{2}\right)$ for all Toeplitz operators in the ball $\mathbb{B}^{2}$.

It is known that

THEOREM 7.4 ([33, 32]).

1. The algebra $\mathcal{A}\left(\mathbb{B}^{2}\right)$ is reflexive.

2. The subspace $\mathcal{T}\left(\mathbb{B}^{2}\right)$ is transitive.

Moreover, we obtain the following from [10] and [Corollary 2.13 and 2.14, 21].

THEOREM 7.6. The algebra $\mathcal{A}\left(\mathbb{B}^{2}\right)$ is hyperreflexive.

Now we can pose

Problem 7.5. How can one characterize the reflexive and hyperreflexive subspaces of $\mathcal{T}\left(\mathbb{B}^{2}\right)$ ? 
8. Isometries, quasinormal operators and power partial isometries. In [34] Sarason also proved that

THEOREM 8.1 ([34]). The algebra generated by a normal operator is reflexive.

After Theorem 6.1 saying that the algebra generated by the unilateral shift is reflexive and Theorem 8.1, a natural question concerns the reflexivity of an isometry. Each isometry is an orthogonal sum of a unilateral shift and a unitary operator. Although the behavior of reflexivity under orthogonal sums (see [36]) is not straightforward, we have

THEOREM 8.2 ([19]). Let $V$ be an isometry. Then the algebra $\mathcal{W}(V)$ is reflexive.

This result was generalized to quasinormal operators.

THEOREM 8.3 ([35]). Let $T$ be a quasinormal operator. Then the algebra $\mathcal{W}(T)$ is reflexive.

Recently it was improved to hyperreflexivity.

THEOREM 8.4 ([25]). Let $T$ be a quasinormal operator. Then the algebra $\mathcal{W}(T)$ is hyperreflexive and $k_{\mathcal{W}(T)}<259$.

Partial isometries are natural generalizations of isometries. Recall that an operator $W \in L(\mathcal{H})$ is a partial isometry if $W^{*} W$ is an orthogonal projection or equivalently $W W^{*} W=W$. An operator $V$ is a power partial isometry if $V^{n}$ is a partial isometry for all positive integers $n$. A simple Jordan block in a finite-dimensional Hilbert space is a power partial isometry. Thus we cannot expect that all power partial isometries are reflexive.

We have the following full characterization of reflexivity of power partial isometries.

TheOREM 8.5 ([5]). Let $V$ be a power partial isometry. Any of the following conditions is sufficient for $V$ to be reflexive:

1. $V^{k+1} V^{*+1} \supsetneqq V^{k} V^{* k}$ for all $k \geqslant 1$,

2. $V^{* k+1} V^{k+1} \supsetneqq V^{* k} V^{k}$ for all $k \geqslant 1$,

3. $V^{k} V^{* k}=V^{k+1} V^{*+1}$ for all $k \geqslant 1$ or $V^{* k} V^{k}=V^{* k+1} V^{k+1}$ for all $k \geqslant 1$,

4. there exists $k \geqslant 2$ such that $V^{k} V^{* k}=V^{k+1} V^{* k+1}$ and

$\operatorname{dim} \mathcal{R}\left(V^{k_{0}-1} V^{* k_{0}-1}\right) \ominus \mathcal{R}\left(V^{k_{0}} V^{* k_{0}}\right)+\operatorname{dim} \mathcal{R}\left(V^{k_{0}-2} V^{* k_{0}-2}\right) \ominus \mathcal{R}\left(V^{k_{0}-1} V^{* k_{0}-1}\right) \geqslant 3$,

where $k_{0}=\inf \left\{k: V^{k} V^{* k}=V^{k+1} V^{* k+1}\right\}$,

5. there exists $k \geqslant 2$ such that $V^{* k} V^{k}=V^{* k+1} V^{k+1}$ and

$\operatorname{dim} \mathcal{R}\left(V^{* k_{0}-1} V^{k_{0}-1}\right) \ominus \mathcal{R}\left(V^{* k_{0}} V^{k_{0}}\right)+\operatorname{dim} \mathcal{R}\left(V^{k_{0}-2} V^{* k_{0}-2}\right) \ominus \mathcal{R}\left(V^{k_{0}-1} V^{* k_{0}-1}\right) \geqslant 3$,

where $k_{0}=\inf \left\{k: V^{k} V^{* k}=V^{k+1} V^{* k+1}\right\}$.

Moreover, if none of the above conditions is fulfilled then $V$ is not reflexive.

In [7] it was shown that reflexivity for large classes of operators can be characterized using rank two operators. A similar characterization can be made for power partial isometries.

THEOREM 8.6. Let $S$ be a completely non-unitary power partial isometry. Then the following are equivalent: 
1. $\mathcal{W}(S)$ is reflexive,

2. one of the conditions $1-5$ of Theorem 8.5 is fulfilled,

3. for any operator $B \in \mathcal{W}(S)$ of rank two, $B$ generates a one-dimensional ideal.

We can put forward the following

Problem 8.7. How can one characterize the reflexivity for partial isometries?

9. Final remarks. It has not been possible to present all reflexivity and hyperreflexivity results in this article since the subject is very large. For example we have not discussed results obtained by the Dual Algebra Technique which has given many interesting results on invariant subspaces and reflexivity. We recall only a few papers where the reader can find the most interesting results and references to other results $[12,11,13,16,26,1]$. A hyperreflexivity result obtained by this technique was given in [10]. Isometries with orthogonal ranges were considered in [18]. In [23] a more abstract definition of reflexivity and hyperreflexivity was presented.

\section{References}

[1] E. Albrecht and M. Ptak, Invariant subspaces for doubly commuting contraction with rich Taylor spectrum, J. Operator Theory 40 (1998), 373-384.

[2] C. Apostol, L. Fialkow, D. Herrero and D. Voiculescu, Approximation of Hilbert Space Operators, Volume II, Research Notes in Mathematics 102, Marshfield, Mass., 1984.

[3] W. B. Arveson, Interpolation problems in nest algebras, J. Funct. Anal. 20 (1975), 208-233.

[4] E. A. Azoff, On finite rank operators and preannihilators, Mem. Amer. Math. Soc., 357 (1986).

[5] E. A. Azoff, W. S. Li, M. Mbekhta and M. Ptak, Power partial isometries, consistent operators and reflexivity, preprint.

[6] E. A. Azoff and M. Ptak, A dichotomy for linear spaces of Toeplitz operators, J. Funct. Anal. 156 (1998), 411-428.

[7] E. A. Azoff and M. Ptak, On rank two linear transformations and reflexivity, J. London Math. Soc. (2) 53 (1996) 383-396.

[8] E. A. Azoff and H. A. Shehada, Literal embeddings of linear spaces of operators, Indiana Univ. Math. J. 42 (1993), 571-589.

[9] M. Bakonyi and D. Timotin, On a conjecture of Cotlar and Sadosky on multidimensional Hankel operators, C. R. Acad. Sci. Paris, Serie I 325 (1997), 1071-1075.

[10] H. Bercovici, Hyper-Reflexivity and the Factorization of linear functionals, J. Funct. Anal. 158 (1998), 242-252.

[11] H. Bercovici, C. Foiaş and C. Pearcy, Dual Algebras with Applications to Invariant Subspaces and Dilation Theory, CBMS Regional Conf. Ser. in Math. 56, Amer. Math. Soc., Providence, 1985.

[12] S. Brown, Some invariant subspaces for subnormal operators, Integral Equations Operator Theory 1 (1978), 310-330.

[13] S. Brown, B. Chevreau and C. Pearcy, On the structure of contraction operators, II, J. Funct. Anal. 76 (1988), 30-55.

[14] J. B. Conway, A Course in Operator Theory, American Mathematical Society, Providence, 2000 . 
[15] J. B. Conway and M. Ptak, The harmonic functional calculus and hyperreflexivity, Pacific J. Math. 204 (2002), 19-29.

[16] B. Chevreau, G. Exner and C. Peacy, On the structure of contraction operators, III, Michigan Math. J. 36 (1989), 29-62.

[17] K. Davidson, The distance to the analytic Toeplitz operators, Illinois J. Math. 31 (1987), 265-273.

[18] K. Davidson and D. Pitts, Invariant subspaces and hyper-reflexivity for free semigroup algebras, Proc. London Math. Soc. (3) 78 (1999), 401-430.

[19] J. A. Deddens, Every isometry is reflexive, Proc. Amer. Math. Soc 28 (1971), 509-511.

[20] J. A. Deddens and P. A. Fillmore, Reflexive linear transformations, Linear Algebra Appl. 10 (1975), 89-93.

[21] J. Eschmeier, On $C_{00}$-representations of $H^{\infty}(G)$ with dominating Harte spectrum, in: Banach Algebras '97 (Blaubeuren), de Gruyter, Berlin, 1998, 135-151.

[22] S. H. Ferguson, The Nehari problem for the Hardy space on the torus, J. Operator Theory 40 (1998), 309-321.

[23] D. Hadwin, A general view of reflexivity, Trans. Amer. Math. Soc. 344 (1994), 325-360.

[24] L. Kerchy, Reflexive subspaces of generalized Toeplitz operators, Acta Sci. Math., to appear.

[25] K. Kliś and M. Ptak, Quasinormal operators are hyperreflexive, in: Banach Center Publications 67, Institute Mathematics, Polish Academy of Sciences, 2005, 241-244.

[26] M. Kosiek and M. Ptak, Reflexivity of $N$-tuples of contractions with rich joint left essential spectrum, Integral Equations Operator Theory 13 (1990), 395-420.

[27] J. Kraus and D. Larson, Reflexivity and distance formulae, Proc. London Math. Soc. 53 (1986), 340-356.

[28] A. Loginov and V. Shulman, Hereditary and intermediate reflexivity of $W^{*}$ algebras, Math. USSR-Izv. 9 (1975), 1189-1201.

[29] B. Magajna, On the distance to finite-dimensional subspaces in operator algebras, J. London Math. Soc. 47 (1993), 516-532.

[30] V. Müller and M. Ptak, Hyperreflexivity of finite-dimensional subspaces, J. Funct. Anal. 218 (2005), 395-408.

[31] M. Ptak, On the reflexivity of the pairs of isometries and of tensor products of some operator algebras, Studia Math. 83 (1986), 47-53.

[32] M. Ptak, Reflexivity of multiplication operators in certain domains in $\mathbb{C}^{N}$, Bull. Acad. Pol. Sci., Ser. Math. 37 (1989), 217-220.

[33] M. Ptak, On the reflexivity of multigenerator algebras, Dissertationes Mathematicae 378 (1998).

[34] D. Sarason, Invariant subspaces and unstarred operator algebras, Pacific J. Math. 17 (1966), 511-517.

[35] W. R. Wogen, Quasinormal operators are reflexive, Bull. London Math. Soc 11 (1979), $19-22$.

[36] W. R. Wogen, Some counterexamples in nonselfadjont algebras, Annals of Mathematics 126 (1987), 415-427. 
